\title{
Deconstructive versus reconstructive endovascular approaches for intracranial dissecting aneurysms
}

\author{
Mohammad Ahmad Mohammad Saad ${ }^{1 *}$ (D), Wael Mohammad Fathy Salamah², \\ Maged Abdel Gawwad Abdel Salam', Ashraf Abdel Latif Osman³ and Farouk Hassan ${ }^{4}$
}

\begin{abstract}
Background: The annual incidence of the intracranial dissecting aneurysms is about 1 to 1.5 per 100,000. This is a well-known cause of stroke and subarachnoid hemorrhage in young and middle-aged patients (Santos-Franco et al. in Neurosurg Rev, 2008. https://doi.org/10.1007/s10143-008-0124-x). Various surgical and endovascular treatment methods have been proposed for intracranial dissecting aneurysms. All treatment methods aim to reduce the blood flow in the dissected region. Deconstructive techniques sacrifice the parent artery, whereas reconstructive techniques aim to maintain a parent artery (Stéphanie et al. in Lancet Neurol 14(6):640-654, 2015. https://doi.org/10.1016/S14744422(15)00009-5). Due to its dissecting nature, wall friability can make surgical clipping difficult and even risky. On the other hand, recanalization after coiling alone is almost certain. Therefore, deconstructive modalities of treatment like trapping or parent vessel occlusion, performed either surgically or endovascularly, have predominated for managing those lesions, usually with good results. Nevertheless, in absence of efficient collateral pathways, the deconstructive technique carries an ischemic risk. In situations in which parent artery preservation is mandatory, the use of stentassisted techniques may be the most appropriate choice (de Barros Faria et al. in Am J Neuroradiol 32(11):2192-2195, 2011. https://doi.org/10.3174/ajnr.A2671). However, the usage of stent with recently ruptured aneurysms is always perplexing due to the necessity of dual antiplatelet administration. Hence the management of dissecting aneurysms remain challenging.
\end{abstract}

Results: Between January 2017 and July 2019, 19 patients presenting with intracranial dissecting aneurysms were referred to our department for endovascular treatment. Among the nineteen patients, 11 cases were treated by parent artery occlusion representing $57.9 \%$ of the cases, and 7 cases (36.8\%) were treated by artery preserving technique, and only one case (5.3\%) was treated by combination of parent artery occlusion and artery preserving technique. One week after the intervention $26.3 \%$ of patients had no disability $(\mathrm{mRS}=0), 47.4 \%$ had no significant disability $(\mathrm{mRS}=1)$, $15.8 \%$ had slight disability (mRS $=2$ ) and $10.5 \%$ had moderate to severe disability (mRS $=3-4)$. After three months we found an overall improvement of the clinical outcome, as $57.9 \%$ of patients had no disability (mRS $=0$ ), $26.3 \%$ had no significant disability ( $m R S=1)$ and $15.8 \%$ had mild disability $(m R S=2)$. Finally, after six months reassessment with angiography showed that $89.5 \%$ of patients had stable aneurysmal occlusion, and $10.5 \%$ had recurrence of aneurysm.

\footnotetext{
*Correspondence: saadams71@yahoo.com

${ }^{1}$ Radiology Department, Faculty of Medicine, Fayoum University, Faiyum,

Egypt

Full list of author information is available at the end of the article
} 
Conclusions: Both endovascular approaches, whether parent artery occlusion or artery preserving technique showed favorable outcome which indicates the safety and efficacy of both approaches if selected properly according to the morphology of the aneurysm.

Keywords: Intracranial arterial dissecting aneurysm, Digital subtraction angiography, Parent artery occlusion, Artery preserving techniques

\section{Background}

Arterial dissections are characterized by the sudden disruption of the endothelium, the intima, and the internal elastic lamina with subsequent penetration of circulating blood into the media resulting into arterial narrowing or occlusion with consequent ischemic sequelae or resulting into sub-adventitial aneurysmal formation with possible consequent hemorrhagic event that may occur in children, young and middle-aged adults $[1,2]$.

The absence of an external elastic membrane and the presence of thin muscular and adventitial layers make intracranial arteries potentially prone to sub-adventitial dissection and subsequent subarachnoid hemorrhage. The hemorrhagic presentations occur in $20 \%$ of internal carotid artery dissections and 50\% of vertebral artery dissections. A re-bleeding rate of more than $40 \%$ within the first $24 \mathrm{~h}$ has been reported [3].

The gold standard in vascular imaging is still represented by the digital subtraction angiography (DSA). Classical findings are pearl-and-string sign, double lumen, or a simple fusiform dilatation, in addition to a delayed clearance of the dilatation or a false lumen [4]. MR imaging techniques may sometimes replace the conventional angiography in diagnosis of arterial dissections, due to good resolution nowadays and actual demonstration of the intramural hematoma [5].

Dissecting aneurysms of the intracranial circulation are uncommon, accounting for $3 \%$ of all cerebral aneurysms. They are significantly less frequent than saccular lesions but usually more difficult to treat, with a devastating natural history and up to $80 \%$ mortality in 5 years if left untreated. Dissecting aneurysms pose a great challenge to both surgical and endovascular treatments due to their location and anatomic configuration. The high rate of re-bleeding among patients treated conservatively argues for treatment in the acute phase. Additionally, it is uncommon for a dissecting aneurysm to resolve spontaneously or with medical treatment only, as sometimes seen with arterial dissections. Given this scenario, despite sharing the same etiology, arterial dissection and dissecting aneurysm respond differently to treatment and should be managed accordingly [5].

Various surgical and endovascular treatment methods have been proposed for intracranial dissecting aneurysms. All treatment methods aim to reduce blood flow in the dissected segment. Deconstructive techniques sacrifice the parent artery, whereas reconstructive techniques aim to maintain a parent artery. Parent artery occlusion is a deconstructive technique in which blood flow into the dissected segment of the artery is stopped by occlusion either surgically or through an endovascular approach. Preferably, the dissected segment is occluded both proximally and distally to prevent re-rupture through retrograde filling of a dissecting aneurysm. Parent artery occlusion has a risk of brain infarct in case of insufficient collateral supply. Before permanent occlusion, the collateral supply can be assessed with temporary balloon-occlusion with simultaneous monitoring of the patient's neurological function.

Artery preserving techniques by selective aneurysmal sac occlusion through clipping (surgical) or coiling (endovascular), are often difficult in intracranial dissecting aneurysms given the non-saccular shape of the dissecting aneurysm. In endovascular treatment, stent assisted coiling may be an appropriate option to promote arterial wall healing.

Seldom, a bypass surgery between extracranial and intracranial arteries can be considered if the risk of infarction due to parent artery occlusion is unacceptably high and stenting is impossible. In very rare instances, mostly in middle-cerebral artery dissections (M2 branches), the dissected segment can be excised and arterial stumps re-anastomosed [1].

Because dissecting aneurysms have a pseudo-aneurysm nature, direct microsurgical clipping is usually not feasible. Among the different causes of morbidity and mortality, or reasons for surgical failure, some authors suggest that thrombosis of the tiny perforating branches during surgical trapping contributes to the poor outcomes [3].

So, the introduction of endovascular methods has added an attractive, minimally invasive therapeutic approach and spares the patient some of the hazards associated with craniotomy and open surgery [6]. Also, Endovascular methods can overcome the anatomical limitations using flow diverting stents (FDSs) which are specially designed to reduce flow velocity in the 
aneurysm sac and promote thrombosis while maintaining flow in the main artery and branch vessels [7].

\section{Methods}

Between January 2017 and July 2019, 19 patients presenting with intracranial dissecting aneurysms were referred to our department for endovascular treatment. The patients were subjected to digital subtraction angiography in our Interventional Radiology department and dissecting intracranial aneurysms were managed using endovascular approach. Patients were followed up at 1 week as well as at 3 months and 6 months in the neurology or neurosurgery outpatient clinic and 6 month angiogram follow up was done in the radiology department. The local ethical committee approved this prospective study and written informed consent was taken.

- If stent-assisted coiling or flow diverter stent deployment was planned, antiplatelet therapy was necessary:

- In acutely ruptured aneurysms: Aspirin $300 \mathrm{mg}$ and Ticagrelor $180 \mathrm{mg}$ administered in gastroesophageal ryle after induction of general anaesthesia and half an hour before stent deployment.

- In elective stenting: Aspirin $75 \mathrm{mg}$ once daily and Ticagrelor $90 \mathrm{mg}$ twice daily for 5-7 days before the procedure.

If parent vessel sacrifice was planned, we had to evaluate the collateral supply through the circle of Willis and/ or the lepto-meningeal (pio-pial) collaterals by diagnostic angiography, and balloon occlusion test if needed. In balloon occlusion test, we considered that there is sufficient collateral supply if there was less than $2 \mathrm{~s}$ delay in the venous filling between the tested territory and the contralateral side. In other cases, without doing balloon occlusion test, slow dampened flow in the artery distal to the aneurysm was considered an evidence that this relative hypoperfusion distal to the aneurysm led to development of pio-pial collaterals that was confirmed angiographically by retrograde filling of its branches during injection of other arteries.

In all patients where the parent artery was sacrificed, we either occluded proximal to the aneurysm, or trapped the neck or coiled the sac then occluded the vessel after that. Final angiographic images were obtained to insure total and complete occlusion of the parent vessel and the aneurysm and reconfirm sufficient cross filling from the collateral pathways.

In the cases in which a flow diverter stent was deployed, we used Surpass ${ }^{\mathrm{TM}}$ Streamline $^{\mathrm{TM}}$ (Stryker Neurovascular,
Fremont, CA, USA). In another case, we used the telescopic implantation of two low-profile Braided LVIS Jr. ${ }^{\circledR}$ (MicroVention, Tustin, CA, USA) stents to achieve flow diversion.

Final angiographic images were obtained to insure good placement and apposition of the stent within the parent artery wall and contrast stagnation within the aneurysmal sac.

Clinical follow up was closely done for one month following the procedure. Any clinical event during or within 1 month after treatment, minor or major, transient or permanent, which might be even remotely associated with the procedure, was considered to be procedure related.

Functional outcome was measured and graded according to the modified Rankin scale (mRS 0-6).

Angiographic follow up was done 6 months after the procedure to assess the stability of aneurysmal occlusion or any recurrence occurred.

\section{Statistical analysis}

Patient demographics, pretreatment clinical status, coexisting diseases, treatment methods, post-treatment recurrence and outcomes of prognosis were recorded. The characteristics of the aneurysm, including its location and size, were also analyzed. Data were collected and coded to facilitate data manipulation and double entered into Microsoft Access and data analysis was performed using Statistical Package of Social Science (SPSS) software version 18 in windows 7 . Simple descriptive analysis in the form of numbers and percentages for qualitative data, and arithmetic means as central tendency measurement, standard deviations as measure of dispersion for quantitative parametric data.

For qualitative data: Chi square test to compare two of more than two qualitative groups. Mc-Nemar test for paired dependent qualitative data. The $p$ value $\leq 0.05$ was considered the cut-off value for significance.

\section{Results}

Between January 2017 and July 2019, 19 patients presented with intracranial dissecting aneurysms were referred from the neurosurgery clinic to our department for endovascular treatments. The age of the patients ranged from 3 months to 61 years with the mean age of study group about $(43.5 \pm 12.2)$ years old; 14 of the patients were males $(73.7 \%)$ versus 5 female patients (26.3\%).

Regarding clinical presentation, it was found that $68.4 \%$ of patients presented by SAH, $15.8 \%$ presented by mass effect, and $15.8 \%$ presented by isolated headache.

Regarding the location of the dissecting aneurysms, $78.9 \%$ were located at the posterior circulation versus 
21.1\%located in the anterior circulation. Among patients with anterior circulation dissecting aneurysm, 25\% of them involved the anterior cerebral artery, 25\% involved the internal carotid artery and 50\% involved the middle cerebral artery. As regards the posterior circulation lesions, $46.7 \%$ involved the posterior cerebral artery, $20 \%$ involved the vertebral artery, $13.3 \%$ involved the basilar artery, $13.3 \%$ involved the superior cerebellar artery and 6.7\% involved the posterior inferior cerebellar artery.

Regarding size of the dissecting aneurysms, it was found that $52.6 \%$ of lesions were small size aneurysms measuring less than $10 \mathrm{~mm}$ in diameter, $26.3 \%$ were large aneurysms ranging between $11-24 \mathrm{~mm}$, and $21.1 \%$ had giant size lesion ranging between $25-50 \mathrm{~mm}$.

For Treatment methods, 11 cases were treated by parent artery occlusion, representing $57.9 \%$ of the cases, and 7 cases $(36.8 \%)$ were treated with artery preserving technique and only one case $(5.3 \%)$ was treated by combined technique (Table 1).

The aneurysms treated by parent artery occlusion were involving the pericallosal branch of the anterior cerebral artery in 1 case, the posterior cerebral artery in 6 cases, the superior cerebellar artery in 2 cases, the V4 segment of the vertebral artery in 1 case (Fig. 1) and the PICA in 1 case.

The parent artery occlusion was performed by artery and aneurysmal occlusion (42.1\%), aneurysmal trapping $(10.5 \%)$ or proximal occlusion of the parent artery (5.3\%). Whereas the artery preserving technique was performed by stent-assisted coiling from the first session (15.8\%) (Fig. 2), selective aneurysmal coiling in the acute stage followed by stent deployment in another session (15.8\%) (Fig. 3), or flow diverter stent deployment (5.3\%).

The clinical outcome of the patients was assessed via modified Rankin Scale (mRS). One week after the intervention, $26.3 \%$ of patients had no disability $(\mathrm{mRS}=0)$, $47.4 \%$ had no significant disability $(\mathrm{mRS}=1), 15.8 \%$ had slight disability $(\mathrm{mRS}=2)$ and $10.5 \%$ had moderate to severe disability $(\mathrm{mRS}=4)$.

After three months we found an overall improvement of the clinical outcome, as $57.9 \%$ of patients had no disability $(\mathrm{mRS}=0), 26.3 \%$ had no significant disability $(\mathrm{mRS}=1)$ and $15.8 \%$ had mild disability $(\mathrm{mRS}=2)$.
In our study we found that there is no statistically significant difference with $p$ value $>0.05$ between different ways of treatment as regards the clinical outcomes after one week, and three months of intervention, which indicated that clinical outcomes did not depend on the way of treatment (Table 2).

Finally, after six months reassessment with angiography showed that $89.5 \%$ of patients had stable aneurysmal occlusion, and $10.5 \%$ had partial recurrence of the aneurysm.

In our study we found no statistically significant difference with $p$ value $>0.05$ between different ways of treatment as regards the angiographic outcomes after six months of intervention, which indicated that angiographic outcomes did not depend on the approach of treatment (Table 3).

\section{Discussion}

The annual incidence of the intracranial dissecting aneurysms is about 1 to 1.5 per 100,000 . They are a wellknown cause of stroke and subarachnoid hemorrhage in young and middle-aged patients [4].

Intracranial dissecting aneurysms have some clinical and radiological features that differentiate them from both saccular aneurysms and non-aneurysmal arterial dissections [8]. First, the favorable outcome of arterial dissections with medical therapy alone is not observed in dissecting intracranial aneurysms, which tend to keep growing and eventually bleed. Second, ruptured dissecting aneurysms are unstable and have a tendency to re-bleed in the acute phase with up to $70 \%$ of re-bleeds in the first $24 \mathrm{~h}$ thus requiring urgent treatment as the mortality rate of the re-bleeding is nearly $50 \%$ [7]. Due to its dissecting nature, wall friability can make surgical clipping difficult and even risky. On the other hand, recanalization after coiling alone is almost certain. Therefore, deconstructive modalities of treatment like trapping or parent vessel occlusion, performed either surgically or endo-vascularly, have predominated for managing those lesions, usually with good results. However, in situations in which parent artery preservation is mandatory, the use of stent-assisted techniques may be the most adequate choice [5].

Table 1 Comparisons of treatment in different cases presentations

\begin{tabular}{llllll}
\hline Treatment & \multicolumn{2}{l}{ Presentations } & & & S value \\
\cline { 2 - 5 } & SAH & Headache & Mass effect & & \\
& No. (\%) & No. (\%) & No. (\%) & & \\
\hline Parent artery occlusion & $7(53.8 \%)$ & $3(100 \%)$ & $1(33.3 \%)$ & $11(57.9 \%)$ & 0.09 \\
Artery preserving technique & $6(46.2 \%)$ & $0(0 \%)$ & $1(33.3 \%)$ & $7(36.8 \%)$ & NS \\
Combined ttt & $0(0 \%)$ & $0(0 \%)$ & $1(33.3 \%)$ & $1(5.3 \%)$ & \\
\hline
\end{tabular}




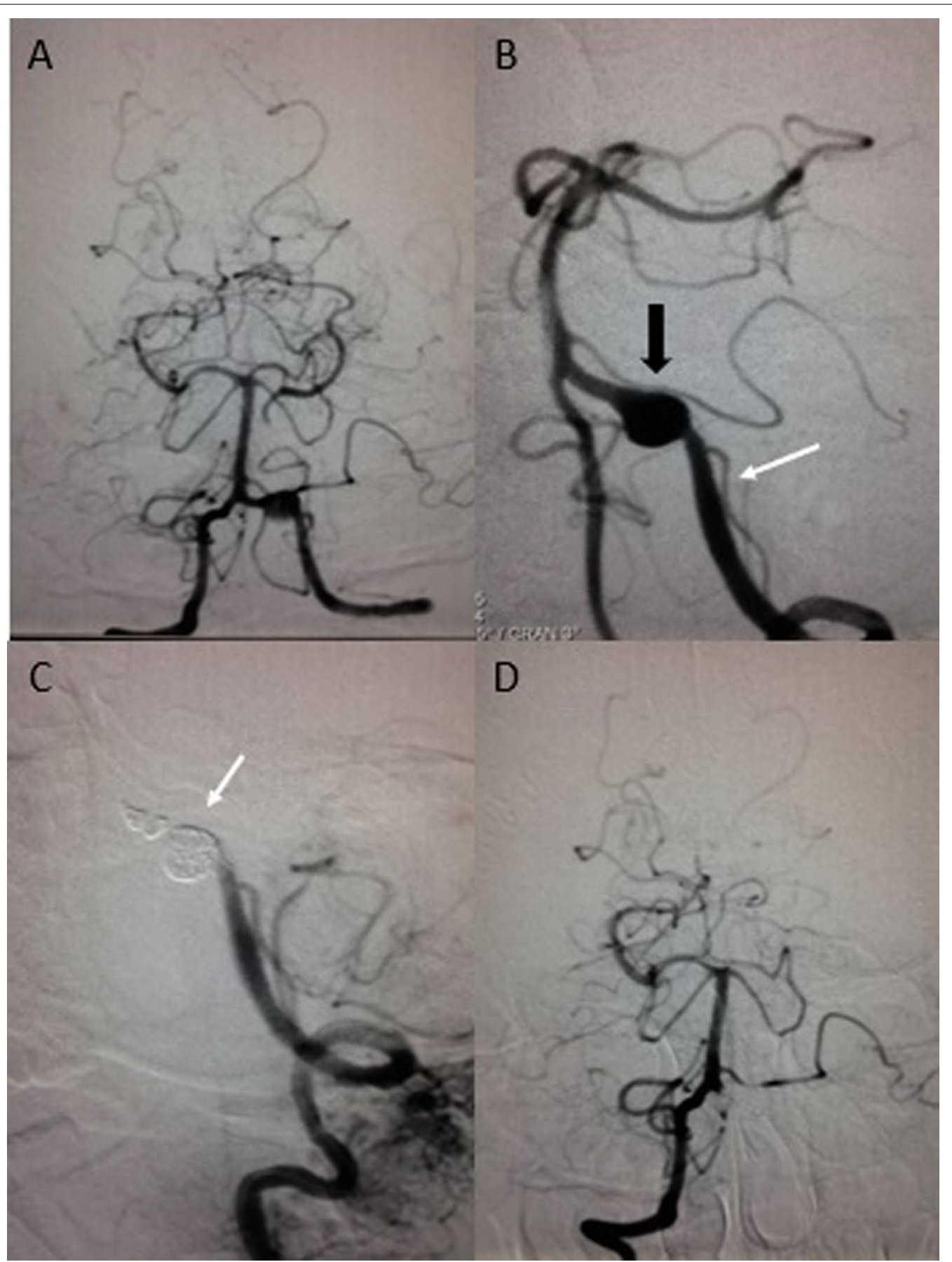

Fig. 1 Conventional angiography showing ruptured left vertebral artery V segment dissecting aneurysm. A Right vertebral artery catherization with retrograde filling of the left vertebral artery and aneurysmal filling. B Left vertebral artery catherization in oblique view, showing the ruptured dissecting aneurysm (black arrow) and the left PICA arising proximal to the aneurysm (white arrow), $\mathbf{C}$ left vertebral artery after occlusion of the aneurysm with parent artery occlusion distal to the origin of the left PICA, coils marked by white arrow, $\mathbf{D}$ right vertebral artery catheterization after coiling with no retrograde filling of the aneurysm

From an angiographic perspective, many aspects in the morphology and location of an aneurysm suggest its dissecting nature: the morphology of an aneurysm being fusiform or irregular in shape, an aneurysm rapidly changing in shape or size, an aneurysm associated with focal stenosis giving the "pearl-and-string" sign, 


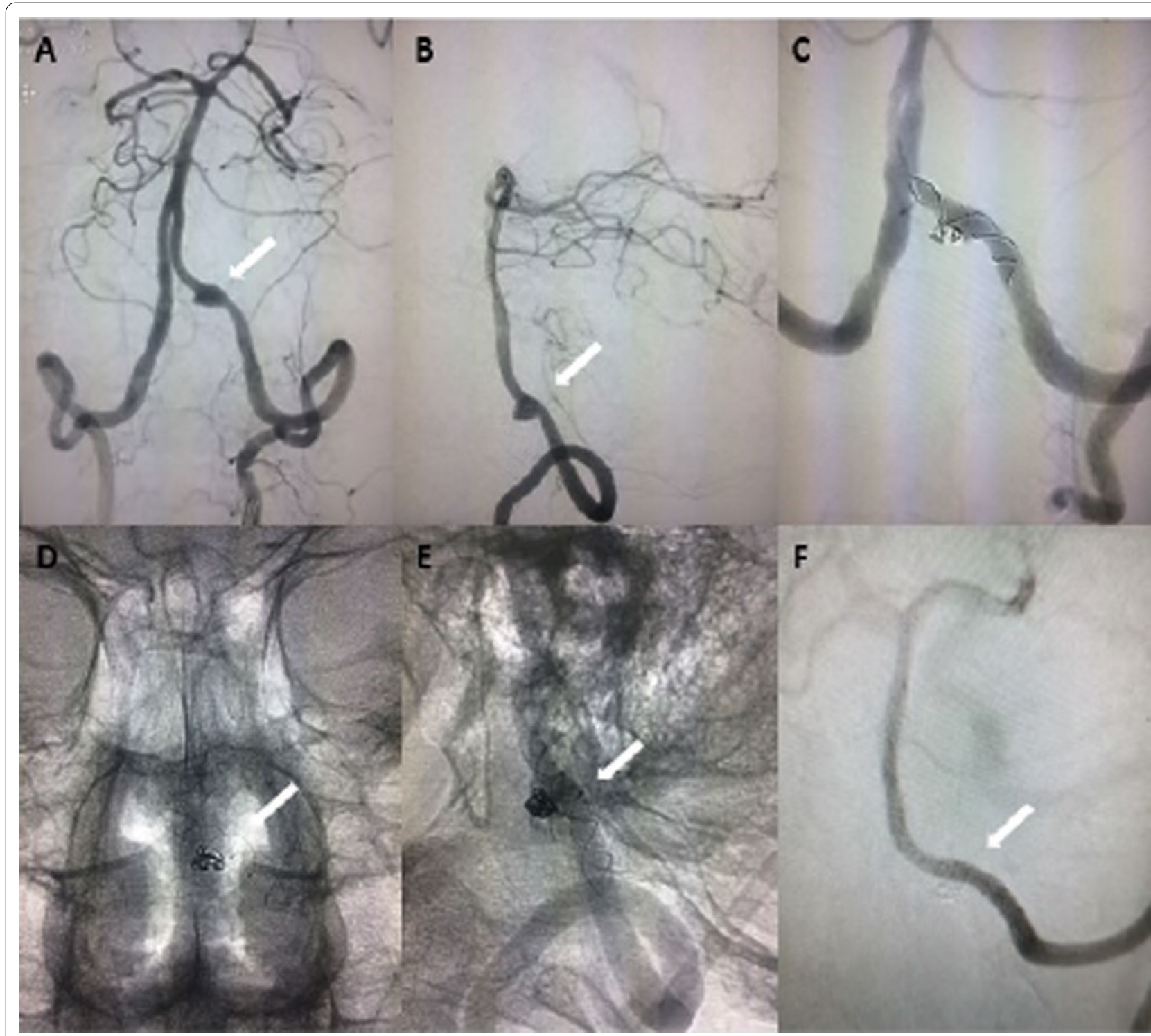

Fig. 2 Stent-assisted coiling of ruptured left vertebral artery V4 segment dissecting aneurysm. Nasogastric tube was inserted under general anaesthesia, followed by administration of loading dose dual antiplatelet 30 min before stent deployment. A, B left vertebral artery catherization, showing the ruptured wide-neck dissecting aneurysm (white arrow), $\mathbf{C}$ coils inserted in the aneurysm sac with braided stent preserving the patency of the artery, D, E non-subtracted images n AP and Lateral views showing the coils and stent (white arrow), $\mathbf{F}$ follow up left vertebral angiography 6 months after the treatment showing stable total occlusion of the aneurysm and patency of the stent (white arrow)

an aneurysm arising at a distal location or from an arterial segment not from the usual bifurcation points, or an aneurysm involving the whole arterial circumference [1].

In our study if the patient presented with ruptured intracranial dissecting aneurysm, the aim of treatment in the acute phase is to secure the patient from re-hemorrhage, so parent artery occlusion is the main line of treatment especially in presence of good collateral supply and no major branches involved. However, in presence of poor collateral supply or major branch included within the dissected segment we tend to use artery preserving techniques, either by securing the aneurysm using coils in the acute stage followed by another step of stent deployment few weeks later to avoid dual antiplatelet in the acute stage or by loading the patient with dual antiplatelet followed by stent-assisted coiling or flow diverter stent deployment.

In subacute stage, according to the location of the aneurysm and the arterial architecture we may choose either artery preserving technique or parent artery occlusion. 

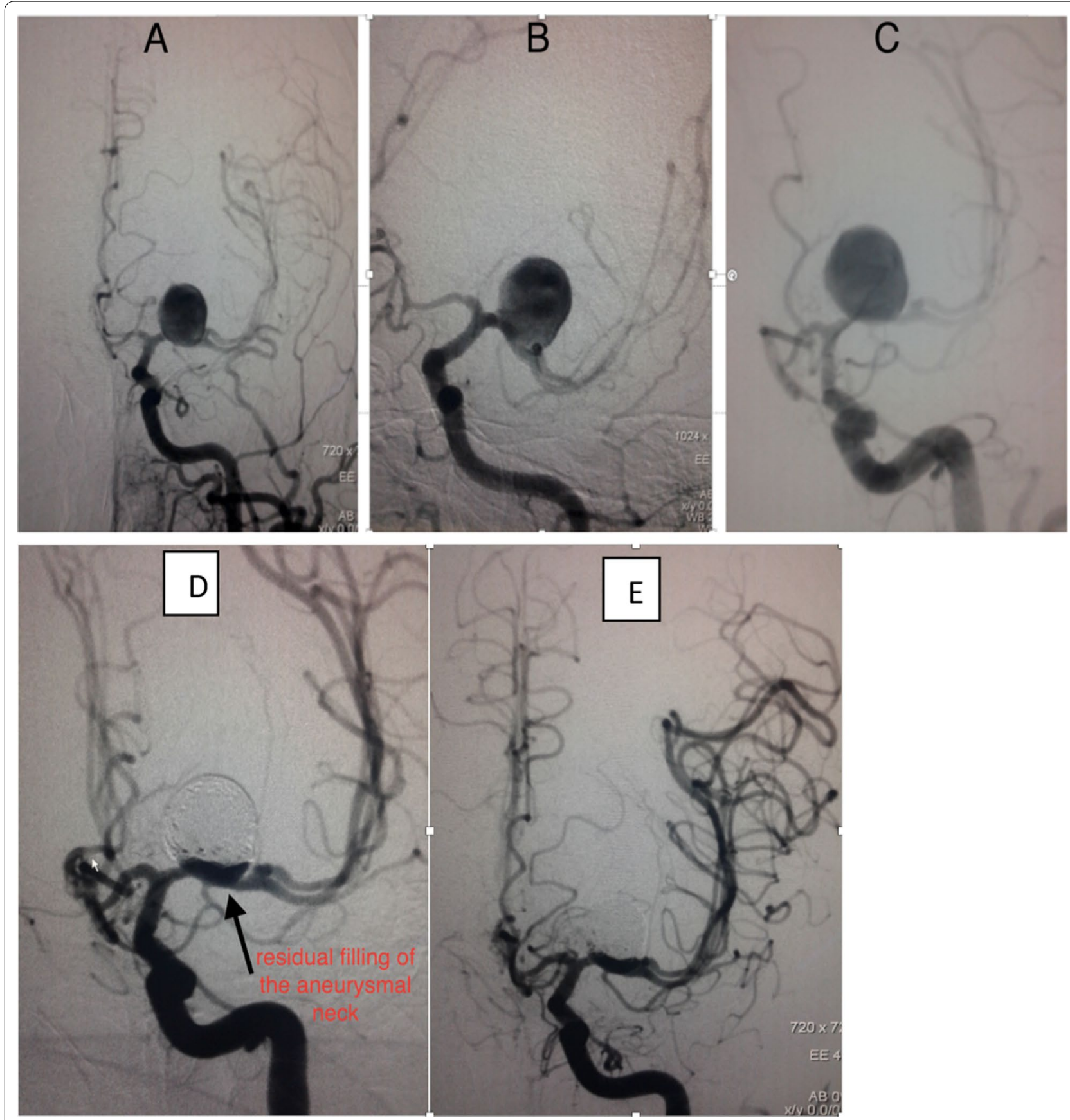

Fig. 3 Conventional angiography A-C showing the left MCA, M1 dissecting aneurysm. Conventional angiography "control film" $\mathbf{D}$, E showing packing of the aneurysm with coils using balloon-assisted coiling in the acute stage, followed by flow diverter stent deployment across the neck of the aneurysm few weeks later

If the patient presented with non-ruptured dissecting aneurysm, we tend to use artery preserving technique if it is technically feasible.

The age of the patients in our study ranged from 3 months to 61 years. The mean age of the study group was $(43.5 \pm 12.2)$ years old. This shows a relative homogeneity with the mean age of intracranial dissecting aneurysm patients published in the literature being $45.6 \pm 8.7$ years and a peak age of onset in the $40 \mathrm{~s}$ [9].

The sex of patients whom presented with dissecting aneurysms in the literature showed a male predominance [10-12]. Our study showed male predominance with 
Table 2 Comparisons of clinical follow up results in different treatment approaches

\begin{tabular}{|c|c|c|c|c|c|}
\hline \multirow[t]{3}{*}{ Follow up } & \multicolumn{3}{|l|}{ Treatment } & \multirow[t]{3}{*}{$p$ value } & \multirow[t]{3}{*}{ Sic } \\
\hline & $\begin{array}{l}\text { Parent artery } \\
\text { occlusion }\end{array}$ & $\begin{array}{l}\text { Artery preserving } \\
\text { technique }\end{array}$ & Combined ttt & & \\
\hline & No. (\%) & No. (\%) & No. (\%) & & \\
\hline \multicolumn{6}{|l|}{ After 1 week (mRS assessment) } \\
\hline No disability (mRS 0) & $3(27.3 \%)$ & $2(28.6 \%)$ & $0(0 \%)$ & 0.97 & NS \\
\hline No significant disability (mRS 1) & $5(45.5 \%)$ & $3(42.9 \%)$ & $1(100 \%)$ & & \\
\hline Mild disability (mRS 2) & $2(18.2 \%)$ & $1(14.3 \%)$ & $0(0 \%)$ & & \\
\hline Moderate to severe disability (mRS 3-4) & $1(9.1 \%)$ & $1(14.3 \%)$ & $0(0 \%)$ & & \\
\hline \multicolumn{6}{|l|}{ After 3 months (clinical assessment) } \\
\hline No disability (mRS 0) & $8(72.7 \%)$ & $2(28.6 \%)$ & $1(100 \%)$ & 0.37 & NS \\
\hline No significant disability (mRS 1) & $2(18.2 \%)$ & $3(42.9 \%)$ & $0(0 \%)$ & & \\
\hline Mild disability (mRS 2) & $1(9.1 \%)$ & $2(28.6 \%)$ & $0(0 \%)$ & & \\
\hline
\end{tabular}

Table 3 Comparisons of angiographic follow up results in different approaches of treatment

\begin{tabular}{|c|c|c|c|c|c|}
\hline \multirow{3}{*}{$\begin{array}{l}\text { After } 6 \text { months (angiographic } \\
\text { assessment) }\end{array}$} & \multicolumn{3}{|l|}{ Treatment } & \multirow[t]{3}{*}{$p$ value } & \multirow[t]{3}{*}{ Sig } \\
\hline & $\begin{array}{l}\text { Parent artery } \\
\text { occlusion }\end{array}$ & $\begin{array}{l}\text { Artery preserving } \\
\text { technique }\end{array}$ & Combined ttt & & \\
\hline & No. (\%) & No. (\%) & No. (\%) & & \\
\hline Stable aneurysmal occlusion & $10(90.9 \%)$ & $6(85.8 \%)$ & $1(100 \%)$ & 0.8 & NS \\
\hline Aneurysmal recurrence & $1(9.1 \%)$ & $1(14.2 \%)$ & $0(0 \%)$ & & \\
\hline
\end{tabular}

male to female ratio $2.8: 1$, among 19 patients 14 of them were males $(73.7 \%)$ versus 5 were females $(26.3 \%)$.

The distribution of the intracranial dissecting aneurysms in our study was $78.9 \%$ located at the posterior circulation versus $21.1 \%$ located in anterior circulation. This is similar to that reported by [12] Kwak et al. who also showed that the prevalence of dissecting aneurysm was $76 \%$ in the posterior circulation [13].

The size of the intracranial dissecting aneurysms was also assessed in our study which showed that $52.6 \%$ of lesions were small size aneurysms, $26.3 \%$ were large aneurysms and $21.1 \%$ had giant size. This is relatively matching with Lee et al. [14] who showed that $68 \%$ of the lesions were small sized aneurysms.

Patients with dissecting aneurysms may present by variable presentations including headache, subarachnoid hemorrhage, neurological deficit due to mass effect or stroke. The reported incidence of SAH in the literature is similar to that of our data. In our series 13 patients (68.4\%) presented by SAH. Yamaura et al. reported that the incidence of SAH was 58\% (206 of 357 cases) [10]. In a study by Mizutani, 108 out of 206 patients treated during the 23 years were admitted with SAH [11]. Also, 86 developed SAH out of 143 patients reported by Ono et al. [12].
Formerly, dissecting aneurysms of the intracranial carotid circulation had been known to cause cerebral infarction in young individuals. However, recent case reports have shown that dissecting aneurysm of the intracranial carotid circulation can cause subarachnoid hemorrhage. Also, Ohkuma et al. [15] revealed that SAH is the dominant type of presentation in the carotid circulation. This is also matching with our results as all patients of the anteriorly located dissecting aneurysm presented with SAH.

Our study showed no statistically significant difference between the different clinical presentations regarding the treatment method. This indicates that the way of endovascular treatment doesn't depend on the presentation symptoms only but also on multiple complicated overlapping factors such as the onset of presentation (e.g. SAH in acute or subacute stage), the site of the lesion (the lesion affecting main artery or small branch, whether proximally or distally located), availability of collateral circulation and vessels condition (e.g. difficult stent deployment in small tortuous vessels).

In the SAH group, 7 patients $(53.8 \%)$ were treated by parent artery occlusion. Whereas 6 patients $(46.2 \%)$ were treated via artery preserving technique. Three of them presented at the subacute stage and were treated by 
stenting (two were treated by stent assisted coiling and one was treated by telescopic stenting using 2 braided stents inducing flow diverter effect to avoid occlusion of the PICA that was arising from the dissected aneurysm of the V4 segment of the vertebral artery). The 3 other patients were treated by selective aneurysmal coiling in the acute stage that was followed by stent deployment few weeks later, to avoid immediate stenting and dual antiplatelet administration in the acute stage. (two of them were affecting the M1 segment of the MCA, so PAO would result in catastrophic outcome, the other one was PCA P2-3 segment small sized aneurysm with preserved normal arterial architecture proximal and distal to it).

Placing coils inside a dissecting aneurysmal sac is not considered alone as a long-term efficient and safe technique. In our study, the 3 patients for whom coils were deployed in the aneurysmal sac at the acute stage, were treated in another session by stent-deployment few weeks later. It was shown in the literature that selective aneurysmal coiling alone in dissecting aneurysms may lead to rebleeding event as described by Krings and Choi [16] due to the weak diseased arterial wall that will lead to aneurysmal regrowth. Hence treatment of a dissecting aneurysm should be performed either by total occlusion of the dissected segment or by stent deployment across the dissected arterial segment to induce endothelialization over the stent mesh, causing healing of the arterial wall.

Among the patients in the non-SAH group; 4 patients representing $66.66 \%$ of the cases were treated by parent artery occlusion, as the aneurysmal size was giant or large, being associated with loss of the normal arterial architecture distal to the dissected segment. One patient $(16.66 \%)$ was treated by artery preserving technique. One patient (16.66) had a vertebro-basilar junction dissecting aneurysm that was treated by combined technique; flow diverter stent was deployed from right vertebral artery to basilar artery then left vertebral artery was occluded distal to the origin of the left PICA to stop the in-flow filling of the aneurysm located at the junction of both vertebral arteries with the basilar artery.

Regarding the complication events in our study, among 11 patients treated with parent artery occlusion, 8 patients showed no significant disability, whereas 3 patients developed post-procedural cerebral infarction. One of them was procedure-related and the 2 other events were sequelae of vasospasm related to the initial subarachnoid hemorrhage. At 3 months follow up only 1 patient kept a mild disability $(\mathrm{mRs}=2)$.

Among 7 patients managed by artery preserving techniques, 5 patients showed no significant disability, whereas 1 patient developed mild symptoms and
1 patient developed moderate disability related to the subarachnoid hemorrhage. At 3 months follow up 2 patients had a mild disability $(\mathrm{mRs}=2)$.

None of our cases who were treated with stent assisted coiling or flow diverter stent showed procedure-related thromboembolic events or delayed aneurysmal rupture. No other technical adverse events occurred such as coil herniation or aneurysmal dome perforation.

Our results show that the final outcome not only depends on the method of treatment whether deconstructive or reconstructive but, it also depends on the neurological hazards related to the presenting insult which in our study was worse within the reconstructive group as some patients developed neurological deficits related to the subarachnoid hemorrhage not to the endovascular management..

Or results are almost matching with the published endovascular studies by Jin et al. [17], Mohammadian et al. [6], Li et al. [18] and Debette et al. [1]. In contrast to the endovascular outcome; Kitanaka et al. reported that the long-term outcome of surgically treated lesions was somehow favorable, but there was a very high rate of postoperative neurological complications, and the major causes of disability were attributable to lower cranial nerve palsy and associated events in half of the patients with poor outcome [19].

Two patients showed partial aneurysmal recurrence at 6 months angiographic follow up. One of them has been treated by parent artery occlusion by coils packed inside a P2 segment dissecting aneurysm of a PCA. Despite packing the whole aneurysmally dilated segment, the dissecting aneurysm showed minimal refilling by retrograde flow from leptomeningeal collaterals to the PCA. The patient was still complaining of headache but no interventional treatment was possible as no endovascular route was amenable to reach the aneurysm again. The patient was kept for symptomatic treatment and follow up. This case showed that in case of parent artery occlusion, deploying coils inside the dissecting aneurysm with parent artery occlusion may be insufficient and it is preferrable to extend the coils to the segment of the parent artery distal to the aneurysm, to avoid back-door refilling of the dissected segment.

The other patient developed partial recurrence of the MCA aneurysm that has been initially treated by stentassisted coiling. The follow up angiogram showed partial aneurysmal refilling within the pack of coils which necessitated another endovascular session passing the microcatheter through the stent struts to add more coils within the aneurysmal sac. 


\section{Conclusion}

Endovascular treatment today plays an important and central role in the overall management of patients with intracranial dissecting aneurysms. In most centers, endovascular treatment is the first-intention approach in the vast majority of patients.

Both treatment methods whether parent artery occlusion or artery preserving technique showed favorable outcome which indicates the safety and efficacy of both approaches if selected properly according to the morphology of the aneurysm.

\begin{abstract}
Abbreviations
ACA: Anterior cerebral artery; AcomA: Anterior communicating artery; AP: Antero-posterior; BA: Basilar artery; CC: Cubic centimeter; CNs: Cranial nerves; CT: Computed tomography; CTA: Computed tomography angiography; DM: Diabetes mellitus; DSA: Digital subtraction angiography; ECA: External carotid artery; FDS: Flow diverter stent; GCS: Glasgow coma scale; ICA: Internal carotid artery; in.: Inch; HTN: Hypertension; IV: Intra-venous; MCA: Middle cerebral artery; ml: Millileter; MRA: Magnetic resonance angiography; mRS: Modified Rankin score; MRI: Magnetic resonance imaging; PA: Postero-anterior; PAO: Parent artery occlusion; PC: Prothrombin concentration; PCA: Posterior cerebral artery; PcomA: Posterior communicating artery; PED: Pipeline Embolization Device; PICA: Posterior inferior cerebellar artery; PT: Prothrombin time; PVO: Parent vessel occlusion; SPSS: Statistical Package of Social Science; TIAs: Transient ischemic attacks; VA: Vertebral artery; Vs: Versus.
\end{abstract}

\section{Acknowledgements}

We acknowledge all patients who involved in the study.

\section{Authors' contributions}

MAS was responsible for the conception, the acquisition and analysis of radiologic findings and revised the final work. WMF participates in the analysis and interpretation of the radiologic data in this study. MS was responsible for the acquisition, analysis and interpretation of the data in this study. AO was responsible for the clinical interpretation of the data in this study. FH was responsible for the design of the work, the interpretation and analysis of radiologic findings and drafted the initial work. All authors read and approved the final manuscript.

\section{Funding}

No source of funds.

\section{Availability of data and materials}

Data available within the article or its supplementary materials.

\section{Declarations}

\section{Ethics approval and consent to participate}

Written consents were taken from all patients accepting to participate in our research work. The local ethical committee, Fayoum University research ethics committee, (committee's reference number is not applicable) approved this retrospective study.

\section{Consent for publication \\ Not applicable.}

\section{Competing interests}

The authors declared that they have no competing interests.

\section{Author details}

${ }^{1}$ Radiology Department, Faculty of Medicine, Fayoum University, Faiyum, Egypt. ${ }^{2}$ Radiology Department, Military Academy, Cairo, Egypt. ${ }^{3}$ Neurosurgery Department, Faculty of Medicine, Fayoum University, Faiyum, Egypt. ${ }^{4}$ Radiology Department, Faculty of Medicine, Cairo University, Giza, Egypt.
Received: 14 May 2021 Accepted: 20 August 2021

Published online: 01 September 2021

\section{References}

1. Stéphanie D, Compter A, Labeyrie M-A, Uyttenboogaart M, Metso TM, Majersik JJ, Goeggel-Simonetti B et al (2015) Epidemiology, pathophysiology, diagnosis, and management of intracranial artery dissection. Lancet Neurol 14(6):640-654. https://doi.org/10.1016/S1474-4422(15) 00009-5

2. Krings T, Piske RL, Lasjaunias PL (2005) Intracranial arterial aneurysm vasculopathies: targeting the outer vessel wall. Neuroradiology 47(12):931-937. https://doi.org/10.1007/s00234-005-1438-9

3. Wong GKC, Poon WS, Chan MTV, Boet R, Gin T, Ng SCP, Zee BCY (2010) Intravenous magnesium sulphate for aneurysmal subarachnoid hemorrhage (IMASH). Stroke 41(10):e576. https://doi.org/10.1161/STROK EAHA.110.589903 (author reply e577)

4. Santos-Franco JA, Zenteno M, Lee A (2008) Dissecting aneurysms of the vertebrobasilar system. A comprehensive review on natural history and treatment options. Neurosurg Rev. https://doi.org/10.1007/ s10143-008-0124-x

5. de Barros Faria M, Nella Castro R, Lundquist J, Scrivano E, Ceratto R, Ferrario A, Lylyk P (2011) The role of the pipeline embolization device for the treatment of dissecting intracranial aneurysms. Am J Neuroradiol 32(11):2192-2195. https://doi.org/10.3174/ajnr.A2671

6. Mohammadian R, Taheraghdam AA, Sharifipour E, Mansourizadeh R, Pashapour A, Shimia M, Shokouhi G, Hashemzadeh A (2013) Endovascular treatment of intracranial artery dissection: clinical and angiographic follow-up. Hindawi Publishing Corporation. https://doi.org/10. 1155/2013/968380

7. Sfyroeras GS, Dalainas I, Giannakopoulos TG, Antonopoulos K, Kakisis JD, Liapis CD (2012) Flow-diverting stents for the treatment of arterial aneurysms. J Vasc Surg 56(3):839-846. https://doi.org/10.1016/j.jvs. 2012.04.020

8. Mizutani T, Kojima H (2000) Clinico-pathological features of nonatherosclerotic cerebral arterial trunk aneurysms. Neuropathology 20(1):91-97. https://doi.org/10.1046/j.1440-1789.2000.00277.x

9. Yamaura A, Watanabe Y, Saeki N (1990) Dissecting aneurysms of the intracranial vertebral artery. J Neurosurg 72(2):183-188. https://doi. org/10.3171/jns.1990.72.2.0183

10. Yamaura A, Ono J, Hirai S (2000) Clinical picture of intracranial nontraumatic dissecting aneurysm. Neuropathology. https://doi.org/10. 1046/j.1440-1789.2000.00276.x

11. Mizutani $T$ (2011) Natural course of intracranial arterial dissections. J Neurosurg 114(4):1037-1044. https://doi.org/10.3171/2010.9.JNS10668

12. Ono $H$, Nakatomi $H$, Tsutsumi $K$, Inoue $T$, Teraoka A, Yoshimoto $Y$, Ide $T$ et al (2013) Symptomatic recurrence of intracranial arterial dissections: follow-up Study of 143 consecutive cases and pathological investigation. Stroke 44(1):126-131. https://doi.org/10.1161/STROKEAHA.112. 670745

13. Kwak JH, Choi JW, Park HJ, Chae EY, Park ES, Lee DH, Suh DC (2011) Cerebral artery dissection: spectrum of clinical presentations related to angiographic findings. Neurointervention 6(2):78-83. https://doi.org/ 10.5469/neuroint.2011.6.2.78

14. Lee JW, Jung JY, Kim YB, Huh SK, Kim DI, Lee KC (2007) Spontaneous dissecting aneurysm of the intracranial vertebral artery: management strategies. Yonsei Med J 48(3):425-432. https://doi.org/10.3349/ymj. 2007.48.3.425

15. Ohkuma H, Suzuki S, Ogane K (2002) Dissecting aneurysms of intracranial carotid circulation. Stroke 33(4):941-947. https://doi.org/10.1161/ 01.STR.0000013564.73522.05

16. Krings T, Choi IS (2010) The many faces of intracranial arterial dissections. Interv Neuroradiol 16(2):151-160. https://doi.org/10.1177/15910 1991001600206

17. Jin SC, Kwon DH, Choi CG, Ahn JS, Kwun BD (2009) Endovascular strategies for vertebrobasilar dissecting aneurysms. Am J Neuroradiol 30(8):1518-1523. https://doi.org/10.3174/ajnr.A1621

18. Li H, Li XF, He XY, Zhang X, Zhu GH, Fang QR, Wang ZQ, Duan CZ (2015) Endovascular treatment of dissecting aneurysms of the posterior 
inferior cerebellar artery and predictors of outcome. J Stroke Cerebrovasc Dis 24(9):2134-2142. https://doi.org/10.1016/j.jstrokecerebrov asdis.2015.05.034

19. Kitanaka C, Eguchi T, Teraoka A, Nakane M et al (1994) Intracranial vertebral artery dissections: clinical, radiological features, and surgical considerations. Neurosurgery 34(4):620-627

\section{Publisher's Note}

Springer Nature remains neutral with regard to jurisdictional claims in published maps and institutional affiliations.
Submit your manuscript to a SpringerOpen ${ }^{\odot}$ journal and benefit from:

- Convenient online submission

- Rigorous peer review

- Open access: articles freely available online

- High visibility within the field

- Retaining the copyright to your article

Submit your next manuscript at $\boldsymbol{\nabla}$ springeropen.com 